Prospective Trial

\title{
Pulsed Radiofrequency Ablation for the Treatment of Glossopharyngeal Neuralgia Secondary to Oropharyngeal Carcinoma
}

Neerja Bharti, DNB ${ }^{1}$, Suryabrata Chattopadhyay, MD¹, Navneet Singla, Mch², Indu Bala, MD¹, Yatindra K Batra, MD ${ }^{1}$, and Jaimanti Bakshi, $\mathrm{MS}^{3}$

From: ${ }^{1}$ Department of Anesthesia \& Intensive Care, Post Graduate Institute of Medical Education and Research, Chandigarh, India;

${ }^{2}$ Department of Neurosurgery, Post Graduate Institute of Medical Education and Research, Chandigarh, India; ${ }^{3}$ Department of Otolaryngology, Post Graduate Institute of Medical Education and Research, Chandigarh, India

Address Correspondence: Neerja Bharti, DNB Professor, Department of Anesthesia \& Intensive Care PGIMER, Chandigarh -160012, India

Email: bhartineerja@yahoo.com

Disclaimer: Pilot study was presented at $14^{\text {th }}$ Annual Pain

Medicine Meeting of ASRA

at Miami, FL, USA. There was no external funding in the preparation of this manuscript. Conflict of interest: Each author certifies that he or she, or a member of his or her immediate family, has no commercial association (i.e., consultancies, stock ownership, equity interest, patent/licensing arrangements, etc.) that might pose a conflict of interest in connection with the submitted manuscript.

Manuscript received: 07-14-2017 Revised manuscript received: 10-23-2017 Accepted for publication: 11-17-2017

Free full manuscript: www.painphysicianjournal.com
Background: The management of glossopharyngeal neuralgia due to oropharyngeal carcinoma is particularly difficult because of rich innervations of the area, erosive nature of neoplasm and dynamic pain evoked by the functional movements like swallowing and chewing. Few case reports have shown the efficacy of pulsed radiofrequency (PRF) treatment in primary and secondary glossopharyngeal neuralgia in non-cancer patients. However, the efficacy of PRF ablation of the glossopharyngeal nerve in oral cancer patients is not known.

Objectives: This study was conducted to evaluate the efficacy and safety of PRF ablation of the glossopharyngeal nerve for the management of oropharyngeal cancer pain involving the base of the tongue, tonsillar fossa, and oropharynx.

Study Design: Prospective interventional clinical trial.

Setting: Tertiary care hospital and medical education and research institute.

Methods: A total of 25 adult patients suffering from oropharyngeal carcinoma, complaining of severe pain in the area mainly supplied by glossopharyngeal nerve, were included. The patients underwent fluoroscopy-guided PRF ablation of the glossopharyngeal nerve of the affected site 3 times at $42^{\circ} \mathrm{C}$ temperature for 120 seconds with 22-gauge, $10 \mathrm{~cm}$ long, $5 \mathrm{~mm}$ active tip radiofrequency needle. They were followed up for one year for pain relief, nausea-vomiting and sleep disturbances. The treatment was considered effective if there was $50 \%$ reduction in pain score at 2 weeks.

Results: There was significant reduction in the overall pain score including site specific pain, odynophagia and ear pain after radiofrequency ablation $(P<0.0001)$ in all the patients. This was associated with decreased opioid consumption $(P<0.001)$, lesser nausea/vomiting, and improved sleep. The treatment was effective in 23 out of 25 patients (92\%) for more than 3 months. No major complication was observed in any patient. The average duration of effective pain relief was 5 to 9 months.

Limitations: Patients having bilateral glossopharyngeal neuralgia or an advanced stage of oral cancer with large lymph nodes at the angle of mandible were not included. This study was a singlecenter observational clinical trial and further multi-center, randomized, controlled trails are needed to obtain higher level of evidence.

Conclusion: Our results showed that PRF ablation can be used effectively and safely for the treatment of glossopharyngeal neuralgia secondary to oropharyngeal carcinoma.

Key words: Glossopharyngeal neuralgia, ear pain, odynophagia, oral cancer pain, pulsed radiofrequency ablation, side effects

Pain Physician 2018; 21:295-301 
G lossopharyngeal neuralgia (GPN) is a rare clinical entity in the general population, though the incidence is high in patients suffering from oropharyngeal malignancy (1). Because the pain is relatively resistant to traditional analgesics, sometimes pain relief can only be achieved at the expense of drug related side effects. Early interventional pain treatment can therefore bypass these overruling side effects and increase the faith of the patient in the pain physician (2). Among invasive interventions, neurolytic nerve blocks, ganglion blocks and even central neuraxial techniques (intrathecal pump) have been tried for intractable oral cancer pain with varying efficacy and adverse effects $(3,4)$.

Pulsed radiofrequency (PRF) ablation which applies short pulse of radiofrequency to neural tissue and has a neuromodulatory type of effect with minimal or no tissue damage is considered as a safe alternative to neurolytic blocks (5). Few case reports have shown the efficacy of PRF ablation in GPN. Shah et al (6) presented the first case report of post tonsillectomy chronic GPN successfully treated with PRF ablation. Another case reports published by Abejon (7) and Chua et al (8) also revealed the efficacy of PRF ablation of glossopharyngeal nerve in non-cancer patients. Among cancer patients, PRF ablation has been effectively used for the treatment of metastatic brachial plexus tumor pain and in head and neck cancer pain $(9,10)$. However, there is a scarcity of studies on PRF application in oral cancer pain management.

Therefore, this prospective clinical trial was conducted to determine the efficacy and safety of PRF ablation of the glossopharyngeal nerve for the management of GPN secondary to oropharyngeal cancer.

\section{Methods}

In this prospective interventional clinical trial, 25 adult patients (18-65 years) suffering from oropharyngeal carcinoma with no curative intervention planned (not fit for surgery), complaining of severe pain in the base of the tongue, tonsillar fossa, and pharynx - area mainly supplied by the glossopharyngeal nerve were included. Patients with unstable cardio-respiratory disorder, coagulation and hematological disorder, psychological disorder or history of drug abuse were excluded. The patients having bilateral GPN and the patients not suitable for intervention due to presence of skin infection or lymph node enlargement at the injection site were also excluded. The institutional ethics committee approval was obtained before starting the trial and written informed consent was taken from all the patients after explaining the procedure. The clinical trial was registered with Clinical Trial Registry [CTRI/2016/08/007179 on 12/08/2016].

The patients were properly assessed regarding site, duration, nature and radiation of pain before the procedure. They were asked to rate their level of perceived pain intensity on a numeric rating scale (NRS) ranging from 0 to 10 , with zero representing no pain and 10 representing the worst pain possible. Apart from site specific pain (base of tongue, tonsillar fossa, angle of mandible), the patients were also evaluated for odynophagia and earache. They ware also assessed for headache, nausea (visual analog scale (VAS) score 0-10, where 0 is no nausea, 10-maximum nausea), vomiting and sleep disturbances (0-10 score where 0- no sleep disturbances and 10- maximum sleep disturbances) (11). Treatment history was taken regarding the use of analgesics (doses, durations, and side effects), other medications, chemotherapy, radiotherapy, and surgery. Physical examination was performed to rule out any systemic illness or local infection at the injection site. The tonsillar fossa and oropharynx were also examined for growth involvement.

\section{PRF of Glossopharyngeal Nerve}

The procedure was performed in the operation theatre under fluoroscopy control on the day care basis. Patients were placed in supine position on the fluoroscopy table and vital parameters - electrocardiography (ECG), heart rate (HR), noninvasive blood pressure (NIBP) and oxygen saturation $\left(\mathrm{SPO}_{2}\right)$ were recorded at the baseline (before procedure) and then at 5 minute intervals until the end of the procedure. Intravenous (IV) access was obtained and mild sedation with IV midazolam (0.5-1 mg) and fentanyl $(50 \mu \mathrm{g})$ was given.

The area of mastoid, lateral neck, and mandible of the affected site was aseptically prepared and draped. Anteroposterior and lateral fluoroscopy images showing styloid process, mastoid and angle of the mandibular ramus were obtained. A subcutaneous skin wheal was raised using 1-2 $\mathrm{mL}$ of $2 \%$ lidocaine at a point overlying midway between the angle of mandible and the mastoid process. A 20-gauge radiofrequency cannula (Cosman Medical Inc., Burlington, USA) was placed about $1.5 \mathrm{~cm}$ deep to the skin aiming to the styloid process in anteroposterior view keeping the tip at the level of the mandibular ramus. A 22-gauge blunt radiofrequency needle, $10 \mathrm{~cm}$ long, $5 \mathrm{~mm}$ active tip was then advanced through the cannula until bony contact 


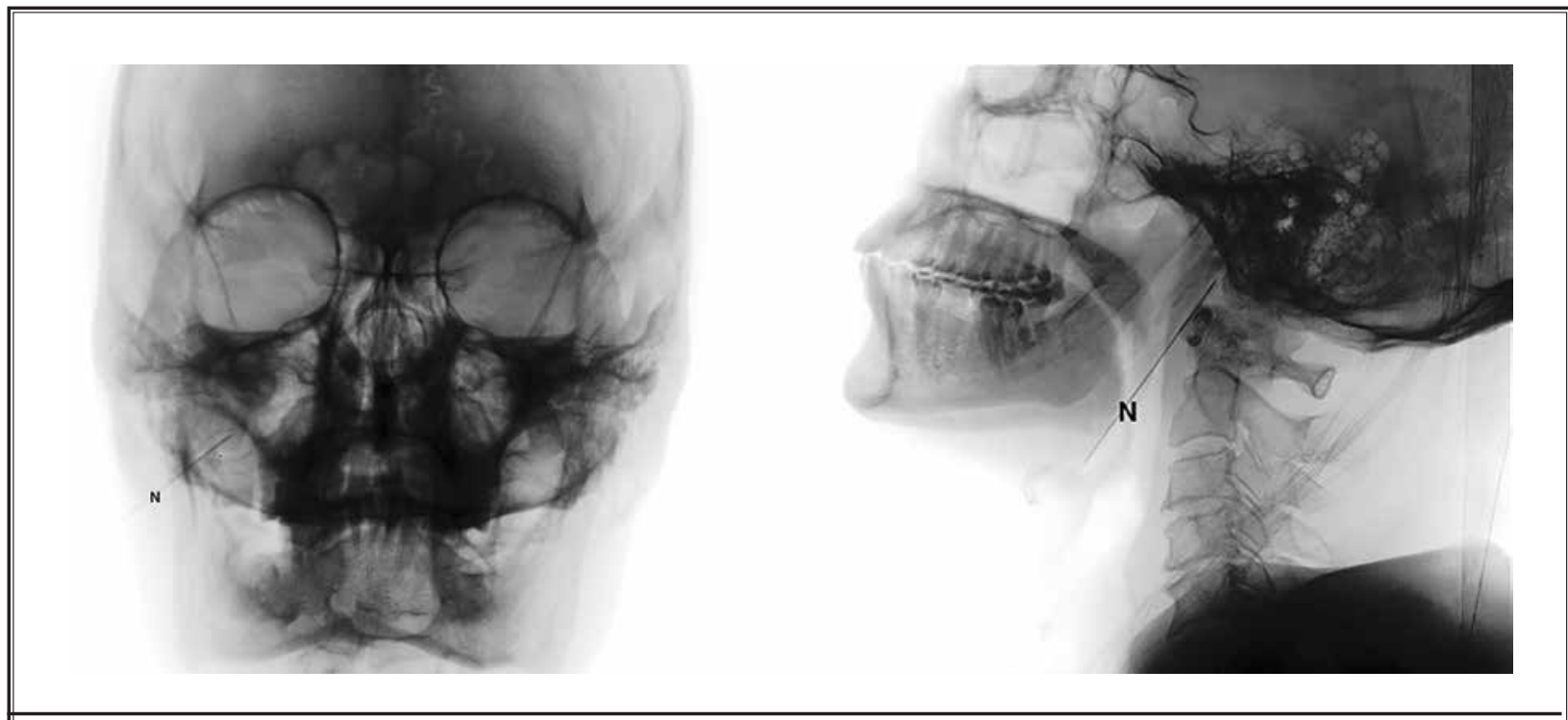

Fig. 1. Fluoroscopic images of needle placement. Left: anterio-posterior vie, Right: lateral view, $n=$ needle.

with the styloid process was made. Then the needle was walked off posteriorly and advanced further $1-1.5 \mathrm{~cm}$. Intermittent O-arm fluoroscopy was used during needle advancement (Fig. 1). Sensory stimulation up to 1.0 volt at $50 \mathrm{~Hz}$ was used to reproduce concordant pain at the base of the tongue, pharynx, and tonsil. The depth and direction of the needle were adjusted according to the patient sensations to ensure correct position. Motor stimulation up to 2.5 volts at $2 \mathrm{~Hz}$ was used to make sure that the contraction of the muscles innervated by the facial, phrenic, and spinal accessory nerve was absent during stimulation. PRF treatment was performed for 3 cycles of 120 seconds at a constant temperature of $42^{\circ} \mathrm{C}$. The rate was $2 \mathrm{~Hz}$ and the pulse rate was $20 \mathrm{~ms}$. Post intervention patients were monitored in the post anesthesia care unit (PACU) for 2 hours for any adverse effect and were also assessed for reduction in pain intensity. The patients were advised to continue same analgesic medications until the next visit (after one week). Thereafter the analgesics were titrated according to the pain intensity.

\section{Post Intervention Patient Assessment}

The patients were assessed weekly for 2 weeks and then monthly at the pain clinic. During their visit all pain scores, including site specific pain, odynophagia and earache, were noted according to NRS. The treatment was considered as effective if there was more than $50 \%$ reduction in overall pain at 2 weeks. The patients were also assessed for reduction in nausea and sleep disturbances. Their satisfaction with the treatment was assessed at the 3 month follow-up at 0-10 points rating score $(0=$ poor, $10=$ excellent $)(12)$.

Statistical analysis was performed using SPSS software version 22.0. Demographic data and hemodynamic parameters ( $\mathrm{HR}$, blood pressure and $\mathrm{SPO}_{2}$ ) were presented as mean and standard deviation. Categorical data were presented as the number of patients and percentages while nonparametric data like pain scores, nausea score, sleep disturbances, patient satisfaction score and Tramadol consumption were presented as median and Inter Quartile Range (IQR). Comparisons of data between different points of time were made by Wilcoxon Signed Rank test. All tests were 2 sided and $P$ $<0.05$ was considered as statistical significant.

\section{Results}

Among 25 patients in the study, 24 were men. The mean age of the study population was $50.76 \pm$ 11.3 years (range: $26-65$ years) and the mean weight was $63.44 \pm 8.36 \mathrm{~kg}$. One patient was diabetic ,controlled with oral hypoglycemic agents and 3 patients were controlled hypertensives. Out of 25 patients, 15 patients $(60 \%)$ were newly diagnosed and 10 patients $(40 \%)$ had a recurrence of carcinoma. Patients were having mainly carcinoma base of tongue (64\%), supraglottic carcinoma (16\%), carcinoma tonsil (8\%), and carcinoma vallecula, oropharynx, hypopharynx each of 
Table 1. Post procedure pain scores (NRS).

\begin{tabular}{|l|c|c|c|}
\hline $\begin{array}{c}\text { Time } \\
\text { interval }\end{array}$ & $\begin{array}{c}\text { Site Specific } \\
\text { Pain }\end{array}$ & Odynophagia & Earache \\
\hline Baseline & $8(7-9)$ & $8(7.5-8)$ & $7(6-8)$ \\
\hline 1 week & $4(3-4)^{*}$ & $4(3-4)^{*}$ & $3(2-3)^{*}$ \\
\hline 2 week & $3(3-4)^{*}$ & $3(3-4)^{*}$ & $2(2-3)^{*}$ \\
\hline 1 month & $3(3-4)^{*}$ & $3(3-4)^{*}$ & $2(2-3)^{*}$ \\
\hline 2 month & $4(3-4) *$ & $3(3-4)^{*}$ & $2(2-3)^{*}$ \\
\hline 3 month & $4(3-4)^{*}$ & $3(3-4)^{*}$ & $2(2-3)^{*}$ \\
\hline
\end{tabular}

Data presented as median (IQR), ${ }^{\star} P<0.0001$ as compared with baseline value

Table 2. Nausea and sleep disturbances during follow-up.

\begin{tabular}{|l|c|c|c|c||}
\hline \multirow{2}{*}{$\begin{array}{l}\text { Follow up } \\
\text { time }\end{array}$} & \multicolumn{2}{|c|}{ Severity of Nausea } & \multicolumn{2}{c|}{ Sleep Disturbances } \\
\cline { 2 - 5 } & VAS Scores & $\boldsymbol{P}$ Value & Scores & $\boldsymbol{P}$ Value \\
\hline Baseline & $5(4-6)$ & & $6(4-6.5)$ & \\
\hline 1 week & $4(3-4.5)$ & 0.005 & $4(3.5-5)$ & $<0.0001$ \\
\hline 2 week & $4(3-4)$ & 0.002 & $4(3-4)$ & 0.001 \\
\hline 1 month & $4(3-4)$ & 0.002 & $4(3-4)$ & 0.001 \\
\hline 2 month & $4(3-4)$ & 0.003 & $4(4-4)$ & 0.001 \\
\hline 3 month & $4(3-4)$ & 0.002 & $4(4-4)$ & 0.001 \\
\hline
\end{tabular}

Data presented as median (IQR)

$4 \%$ in prevalence. They were on oral analgesic medications (Tramadol, Paracetamol, Diclofenac, Pregabalin/ Gabapentin and Amitriptyline), but still complaining of severe pain (NRS $>5$ ). Two patients underwent surgical interventions (wide local excision with modified neck dissection) and 18 had received radio-therapy.

The patients had pain mainly at the area of base of tongue $(76 \%)$, tonsillar fossa $(16 \%)$, and neck ( $8 \%)$ radiating to angle of the mandible or ipsilateral ear $(84 \%)$. The baseline pain score was 8 (7 - 9) [median (Inter Quartile Range)]. Pain was usually throbbing and aching in nature, but some patients also described it as pin prick or burning sensation. The average duration of pain was $2.9 \pm 1.33$ months (range: 1-8 month). All patients complained of odynophagia. Ten patients ( $40 \%)$ also suffered from headache. Most of the patients complained of nausea and sleep disturbances due to pain.

All patients received 3 pulses of PRF treatment of the affected glossopharyngeal nerve and average duration of procedure was $33.6 \mathrm{~min}(20-60 \mathrm{~min})$. No significant variations ( $\pm 20 \%$ of baseline) of the monitored parameters were noted during or after the procedure. Twenty-three among 25 patients (92\%) had effective pain relief with PRF intervention. These patients showed significant reduction in site specific pain, odynophagia and earache $(P<0.0001)$ at each point of time during follow-up (Table 1). They also had significant reduction in nausea and sleep disturbances (Table 2). The other 2 patients developed transient breakthrough pain; one patient experienced it on the 6th and 58th day and, the other patient on the 1st and 8th post PRF day. Average per day Tramadol consumption at 3 months followup was significantly less as compared with baseline [150(100-150) mg, $100(75-100 \mathrm{mg})$ at baseline and 3 months respectively, $P<0.001]$.

No major complication was observed, except 2 patients had transient facial nerve neuropraxia after the procedure, which lasted for 20 minutes and 2 hours, respectively. None of the patient required unplanned admission due to PRF intervention. The patients were satisfied with the treatment and showed overall satisfaction score of 7 (7-9). On long-term follow-up, the mean duration of pain relief was $7.8 \pm 2.6$ months.

\section{Discussion}

Oral cancer is the 8th most common cancer worldwide (13). The incidence of oral cancer is more in men and accounted for more than $30 \%$ of all cancers (14). Anatomical area involved in this cancer is highly sensitive to pain due to rich innervations and the confinement of many anatomical structures to a small space (15). These patients also suffer from odynophagia added with muscular spasm, which limits the mouth opening making it difficult for them to take multiple oral medications for pain relief. So apart from traditional analgesic therapy interventional management may be beneficial for these patients. As oropharyngeal area is mainly supplied by glossopharyngeal nerve, the intervention of glossopharyngeal nerve has the potential of benefiting a major bulk of patients suffering from oral cancer pain.

In the present study, we evaluated the efficacy and safety of PRF ablation of the glossopharyngeal nerve in patients having refractory GPN due to oral cancer. The majority of the patients were men because the habit of chewing and smoking tobacco products is much more prevalent in the male population. The patients were experiencing severe pain mainly at the area involved with the cancerous growth (base of tongue) along with odynophagia. Pain was usually throbbing in nature and radiating to ipsilateral angle of mandible, ear and neck. The PRF ablation of glossopharyngeal nerve of the affected side was performed under all aseptic precautions. The needle placement was easy except in patients who underwent radio therapy previously or had lymph 
node enlargement at the concerned area. During the procedure, at the time of motor stimulation 3 patients showed contractions at facial nerve area and 2 patients showed contractions of spinal accessory nerve (trapezius muscles). In these situations we promptly stopped the motor stimuli, changed the needle position under O-arm guidance and repeated sensory and motor stimuli subsequently. The patients were comfortable during the procedure and no major complication was reported. The pain was significantly reduced $(>50 \%$ reduction in NRS) after the procedure in all the patients.

We found that 23 out of 25 patients had sustained pain relief after the procedure with significant reduction in odynophagia and earache during each followup visit for 3 months. We also observed a significant reduction in Tramadol intake, which may have contributed to reduction of nausea scores in these patients. Sleep disturbances were also significantly diminished when compared with the baseline values. Due to significant pain relief and improvement in sleep, the patients reported high overall satisfaction scores. The 2 patients who did not have sustained pain relief after the procedure, had aggressive local spread of the disease along with painful lymph node enlargement at the submandibular region.

On long-term follow-up, the duration of effective pain relief was around 5-9 months. The pain recurred due to rapid progression of the disease. Most of these patients were not suitable for the repeat procedure due to the presence of large lymph nodes at the site of needle placement. Three patients complained of pain on the other side of the face. In these patients the procedure was repeated on the other side. Two patients died and 3 did not follow-up. Previous case reports also observed early and effective pain relief after PRF treatment in patients with GPN. Abejon (7) presented 2 cases of PRF application for GPN refractory to all treatment approaches. The first case was diagnosed with Hodgkin's lymphoma involving the tonsils manifesting as GPN and the second case was diagnosed with essential GPN. The PRF procedure was effective in both patients leading to significant reduction in pain scores. Overall self reported improvement assessed subjectively 6 months after PRF was 75\% for Hodgkin's lymphoma and $50 \%$ for essential neuralgia. Chua et al (8) observed effectiveness of the procedure in a patient suffering from severe stabbing pain with paroxysm at the back of throat radiating to angle of mandible, upper part of neck, ear and often accompanied by an occipital headache. PRF ablation of the glossopharyngeal nerve resulted in an overall reduction in pain intensity and paroxysm frequency during 4-5 month follow-up. Shah et al (6) reported pain free interval of more than 8 months after PRF treatment of the glossopharyngeal nerve in a patient suffering from GPN due to chronic tonsillitis. The early recurrence of pain in our study may be due to invasive nature of the neoplasm.

A recent retrospective study reported the efficacy of CT-guided radiofrequency thermocoagulation of the glossopharyngeal nerve in 80 patients of GPN; though the procedure was effective in $78.8 \%$ of patients, it was associated with many postprocedural complications including dysesthesias, dysphagia, and diminished gag reflex. Three patients experienced persistent severe dysesthesias of the tongue that impaired their daily life (16).

PRF is a safe and effective modality which has been used in diverse chronic pain conditions but still underused in cancer pain management. PRF has a temperature independent effect on the cell morphology, synaptic transmission, and pain signalling via different pathways including c-Fos gene mediated, by inhibiting excitatory $C$ fibers and by activating transcription factor 3 (ATF3) $(17,18)$. Though PRF has been demonstrated a significant margin of safety in different clinical scenarios due to lack of heat-induced tissue damage, still there is possibility of bleeding, hematoma, infection and neuritis. During PRF treatment of the glossopharyngeal nerve there may be chance of inadvertent puncture of surrounding great vessels (carotid artery and internal jugular vein), inadvertent trauma or block of nerves in proximity namely facial, vagus, hypoglossal and spinal accessory nerves or trauma to the glossopharyngeal nerve itself.

The glossopharyngeal nerve contains both motor and sensory fibers. The sensory fibers supply to the middle ear, posterior third of the tongue, soft palate, tonsillar fossa and upper pharynx. It also innervates the carotid sinus and carotid body. Therefore, GPN can present as odynophagia, earache and upper cervical pain. The motor fibers innervate stylopharyngeus muscle; blockade of motor portion of glossopharyngeal nerve can result in dysphagia secondary to weakness of the stylopharyngeus muscle. Therefore, we performed the procedure under fluoroscopic guidance with hemodynamic monitoring and pre-procedure motor and sensory electrical stimulation.

Various approaches have been used to target the glossopharyngeal nerve percutaneously $(19,20)$. Intraoral approach caries the risk of infection and injury to vital structures including brain stem, vertebral artery 
and upper cervical spinal nerves (19). An extra-oral approach targeting the medial part of the foramen ovale has been demonstrated; however, it can also cause damage to vital neurovascular structures (20). We used the extra-cranial extra-oral approach as described by Shah et al (6), as we found this approach easy and safe. The appropriate size and length of the needle, in particular, the correct exposed tip length is critical in improving the efficacy of the procedure and reducing the risk of inadvertent tissue injury. We used 22-gauge, $10 \mathrm{~cm}$ blunt radiofrequency needles with $5 \mathrm{~mm}$ active tips to reduce post-procedural neuritis and trauma to the surrounding structures. We did not have any major complications except transient facial nerve paresis in two patients just after the procedure. Both the patients recovered spontaneously without any residual effect.

\section{Limitations}

There are some limitations of this study. We excluded the patients having bilateral GPN as we were reluctant to perform bilateral PRF treatment of the glossopharyngeal nerve in the same sitting due to safety concerns. Many patients presenting late with multiple sensory nerve involvement like mandibular and maxillary nerve along with glossopharyngeal nerve were also not included due to difficulty in assessment. Our study was a single-center observational clinical trial. Further, multi-center, randomized controlled trails are needed to provide higher level of evidence about the efficacy of PRF ablation in cancer pain management.

\section{Conclusion}

In conclusion, we found that PRF ablation of glossopharyngeal nerve is an effective and safe procedure for the management of GPN secondary to oral carcinoma. PRF ablation, not only reduced the site specific pain, but also produced reduction in odynophagia, radiating earache, neck pain, nausea, vomiting and sleep disturbances leading to overall improvement in quality of life. The procedure should be contemplated early in these patients as in the late presentation of oropharyngeal cancer hard lymph node enlargement at the angle of mandible is common leading to difficulty in needle placement.

\section{Author Contributions}

Drs. Neerja Bharti and Suryabrata Chattopadhyay had full access to all the data in the study and take responsibility for the integrity of the data and the accuracy of the data analysis. Drs. Neerja Bharti and Navneet Singla designed the study protocol. Dr. Suryabrata Chattopadhyay managed the literature searches and summaries of previous related work and wrote the first draft of the manuscript. Drs. Indu Bala, Yatindra K Batra and Jaimanti Bakshi provided revision for intellectual content and final approval of the manuscript.

\section{Disclaimer}

There was no external funding in the preparation of this manuscript.

\section{Conflict of interest}

Each author certifies that he or she, or a member of his or her immediate family, has no commercial association (i.e., consultancies, stock ownership, equity interest, patent/licensing arrangements, etc.) that might pose a conflict of interest in connection with the submitted manuscript. 


\section{References}

1. Pflendler DF. Glossopharyngeal neuralgia with tongue carcinoma. Arch Otolaryngol Head Neck Surg 1997; 123:658.

2. Bhatnagar S. Individualized interventional pain management technique in early stage of cancer pain: A desirable protocol for improving quality of life. Indian J Palliat Care 2011; 17:91.

3. Datta S, Pai UT. Interventional ap proaches to management of pain of oral cancer. Oral Maxillofac Surg Clin North Am 2006; 18:627-641.

4. Dios PD, Leston JS. Oral cancer pain. Oral Oncology 2010; 46:448-451.

5. Cahana A, Van Zundert J, Macrea L, Van Kleef M, Sluijter M. Pulsed radiofrequency: Current clinical and biological literature available. Pain Med 2006; 7:411-423.

6. Shah RV, Racz GB. Pulsed mode radiofrequency lesioning to treat chronic post-tonsillectomy pain (secondary glossopharyngeal neuralgia). Pain Pract 2003; 3:232-237.

7. Abejon D, García del Valle $S$, Nieto C, Delgado C, Gómez-Arnau JI. Pulsed radiofrequency treatment in idiopathic and secondary glossopharyngeal neuralgia: Preliminary result in 2 cases. $\mathrm{Rev}$ Esp Anestesiol Reanim 2005; 52:109-114.

8. Chua NHL, Beems T, Vissers KC. Two cases of glossopharyngeal neuralgia successfully treated with pulsed radiofrequency treatment. Ann Acad Med 2011; 40:387-389.

9. Rana H, Machett $\mathrm{G}$. Using pulsed radiofrequency ablation to treat pain associated with a tumor involving the brachial plexus. Pain Physician 2013; 16:E311-E314.

10. Bhatnagar S, Rana S, Mishra S. Pulsed RFA of mandibular and maxillary nerve in orofacial pain in advanced head and neck cancer patients - A randomized clinical controlled study. Oral Oncology 2011; 47:565.

11. Elder SJ, Pisoni RL, Akizawa T, Fissel R. Sleep quality predicts quality of life and mortality risk in haemodialysis patients: Results from the Dialysis Outcomes and Practice Pattern Study (DOPPS). Nephrol Dial Transplant 2008; 23:998-1004.

12. Mengelsdroff AD. Patient satisfaction score. Med care 1979; 17:86-90.

13. Petersen PE. The World Oral Health Report 2003: Continuous improvement of oral health in the $21^{\text {st }}$ century - the approach of the WHO Global Oral Health Programme. Community Dent Oral Epidemiol 2003; 31:3-24.

14. Kulkarni MR. Head neck cancer burden in India. Int J Head Neck Surg 2013; 4:29-35.

15. Chua KS, Reddy SK, Lee MC, Patt RB. Pain and loss of function in head and neck cancer survivors. J Pain Symptom Manage 1999; 18:193-202.

16. Wang X, Tang Y, Zeng Y, Ni J. Longterm outcomes of percutaneous radiofrequency thermocoagulation for glossopharyngeal neuralgia: A retrospective observational study. Medicine 2016; 95:e5530.

17. Van Zundert J, de Louw AJ, Joosten EA, Kessels AG, Honig W, Dederen PJ, Veening JG, Vles JS, van Kleef M.. Pulsed and continuous radiofrequency current adjacent to the cervical dorsal root ganglion of the rat induces late cellular activity in the dorsal horn. Anesthesiology 2005; 102:125-131.

18. Hamann W, Abou-Sherif S, Thompson S, Hall S. Pulsed radiofrequency applied to dorsal root ganglia causes a selective increase in $\mathrm{ATF}_{3}$ in small neurons. Eur J Pain 2006; 10:171-176.

19. Raj PP, Lou L, Erdine S, Staats P. Glossopharyngeal nerve block. In: Raj PP, ed. Radiographic Imaging for Regional Anesthesia and Pain Management. Philadelphia, PA, Churchill Livingstone, 2002: pp. 56-6o.

20. Arias M. Percutaneous radio-frequency thermocoagulation with low temperature in the treatment of essential glossopharyngeal neuralgia. Surg Neurol 1986; 25:94-96. 
